\title{
Extended Radical Resection: The Standard of Care for Patients with Advanced Pelvic Malignancy
}

\author{
Michael J. Solomon, MBBCH(Hons), BAO, MSc, DMed, DMedSc, FRCSI, FRACS, FASCRS ${ }^{1,2,3,4}$, and \\ Kilian G. M. Brown, MBBS, BBiomed, M.Phil ${ }^{1,2,3}$ \\ ${ }^{1}$ Surgical Outcomes Research Centre (SOuRCe), Royal Prince Alfred Hospital, Sydney, NSW, Australia; ${ }^{2}$ The Institute of \\ Academic Surgery at RPA, Sydney Local Health District, Sydney, Australia; ${ }^{3}$ Department of Colorectal Surgery, Royal \\ Prince Alfred Hospital, Sydney, Australia; ${ }^{4}$ University of Sydney, Sydney, NSW, Australia
}

In 2019, extended radical resection (or exenterative surgery) represents the standard of care for patients with locally advanced and recurrent malignancies of the pelvis. Although acceptance of these ultra-radical procedures has been slow among surgeons due to the historically high rates of morbidity and mortality reported during the pioneering periods of the 1940s and 1950s, recent decades have seen dramatic improvements in outcomes, particularly for recurrent rectal cancer. These outcomes are now comparable with those of hepatic and pulmonary metastatectomy for primary colorectal cancer. In addition to robust morbidity and long-term survival data, quality-of-life improvements after surgery have now been confirmed by prospective studies. ${ }^{1}$

In this issue of the journal, Peacock et al. ${ }^{2}$ present an analysis of postoperative morbidity from an international collaborative experience with more than 600 patients during 25 years. These impressive results are a welcome addition to the growing literature demonstrating the safety of extended radical resections in the perioperative period. They also reflect a 25-year regional policy in Australia and New Zealand of quaternary referral to a handful of specialized units that have collaborated in research and audit,

(C) Society of Surgical Oncology 2019

First Received: 19 August 2019;

Published Online: 13 September 2019

M. J. Solomon, MBBCH(Hons), BAO, MSc, DMed, DMedSc, FRCSI, FRACS, FASCRS

e-mail: professor.solomon@sydney.edu.au developing new surgical techniques and policies concerning the indications and contraindications for patients with advanced primary and recurrent pelvic malignancies.

The most significant development in exenterative surgery during the last decade has been the redefining of "resectability." Complete oncologic excision (i.e., R0 resection) is the most important predictor of long-term survival, and a clear resection margin has therefore become the "holy grail" for surgeons who perform multi-visceral pelvic resections. On this basis, the lists of contraindications to curative surgery, which were historically based on anatomic boundaries and technical limitations, are no longer relevant in 2019, as increasingly radical resections, such as high sacrectomy, iliac vessel resection, and sciatic nerve excision, are performed routinely and safely at expert units.

The indication for extended radical surgery in contemporary practice is the ability to achieve R0 resection with acceptable morbidity. The paradigm has shifted, and the exenteration surgeon no longer considers what can be technically and safely resected, but what should be resected. It would have been interesting to see the authors' R0 outcomes in this or subsequent publications.

In light of the encouraging data published in the last decade, interest in exenterative surgery globally has been growing, as reflected by the increasing number of both high- and low-volume specialist units and the formation of international collaborations dedicated to improving outcomes for this group of patients. The PelvEx collaborative, ${ }^{3}$ the Beyond TME Collaboration, ${ }^{4}$ and the IMPACT Initiative ${ }^{5}$ have played important roles in 
providing a forum for surgeons to engage with one another and in facilitating the coordinated collection and pooling of data for what remains a relatively uncommon procedure.

As with other complex oncologic surgeries, the high case volume that can be achieved by centralising services at specialized referral centers may improve oncologic and morbidity outcomes while lowering the cost associated with surgery. ${ }^{6}$ It is interesting to note that the vast majority of centers offering exenterative surgery in France and the United Kingdom perform fewer than five procedures per year. $^{7,8}$ Although encouraging outcomes have been achieved in low-volume units, this generally has involved selected patients with centrally based and often primary tumors without pelvic sidewall or sacral involvement. ${ }^{9}$ For patients with locally recurrent rectal cancer and where there is concern about tumor proximity to bone and neurovascular structures at the periphery of the pelvis, surgery should be centralized to high-volume units with a dedicated multidisciplinary surgical team.

Accessibility to surgery for patients with potentially curable pelvic malignancy remains a concern, and more defined referral pathways to high-volume units also may help to ensure that all such patients are able to access surgical teams that routinely operate outside the traditional mesorectal planes.

One of the remaining challenges facing the exenteration community is the lack of an accepted definition for a procedure described more than 70 years ago. Although pelvic exenteration has historically referred to complete evisceration of the pelvis, the techniques of lateral neurovascular and composite bony resection that have evolved since has led the authors' unit to consider pelvic exenteration as a procedure with en bloc resection of a minimum of three pelvic compartments and/or bone or lateral neurovascular resection. Without a standardized definition for these procedures, it is difficult to validly compare outcomes between units or across time periods.
Exenterative surgery has undergone dramatic evolution in recent decades from what was a palliative procedure in gynecologic practice. It now represents the possibility of cure for patients with advanced pelvic malignancy and the standard of care for surgical oncologists.

DISCLOSURE There are no conflicts of interest.

\section{REFERENCES}

1. Steffens D, Solomon MJ, Young JM, et al. Cohort study of longterm survival and quality of life following pelvic exenteration. BJS Open. 2018;2:328-35.

2. Peacock O, Waters PS, Kong JC, Warrier SK, Wakeman C, Eglinton $\mathrm{T}$, et al. Complications after extended radical resections for locally advanced and recurrent pelivc malignancies: a 25 -year experience. Ann Surg Oncol. 2019. https://doi.org/10.1245/s1043 4-019-07816-8.

3. PelvEx Collaborative. Changing outcomes following pelvic exenteration for locally advanced and recurrent rectal cancer. BJS Open. 2019;3:516-20.

4. Beyond TME Collaboration. Consensus statement on the multidisciplinary management of patients with recurrent and primary rectal cancer beyond total mesorectal excision planes. Br J Surg. 2013;100:1009-14.

5. Vallance AE, Harji D, Fearnhead NS, et al. Making an IMPACT: a priority setting consultation exercise to improve outcomes in patients with locally advanced, recurrent, and metastatic colorectal cancer. Eur J Surg Oncol. 2019;45(9):1567-74.

6. Venchiarutti RL, Solomon MJ, Koh CE, Young JM, Steffens D. Pushing the boundaries of pelvic exenteration by maintaining survival at the cost of morbidity. Br J Surg. 2019;106:1393-403.

7. Denost Q, Faucheron JL, Lefevre JH, et al. French current management and oncological results of locally recurrent rectal cancer. Eur J Surg Oncol. 2015;41:1645-52.

8. Harji DP, Griffiths B, McArthur DR, Sagar PM. Current UK management of locally recurrent rectal cancer. Colorectal Dis. 2012;14:1479-82.

9. Humphries EL, Kroon HM, Dudi-Venkata NN, Thomas ML, Moore JW, Sammour T. Short- and long-term outcomes of selective pelvic exenteration surgery in a low-volume specialized tertiary setting. ANZ J Surg. 2019;89:E226-E230.

Publisher's Note Springer Nature remains neutral with regard to jurisdictional claims in published maps and institutional affiliations. 